\title{
Doença de Parkinson: Padrão epidemiológico de internações no Brasil
}

\author{
Parkinson's Disease: Epidemiological pattern of hospital admission \\ Enfermedad de Parkinson: Patrón epidemiológico de admisiones em Brasil
}

Recebido: 08/12/2021 | Revisado: 13/12/2021 | Aceito: 21/12/2021 | Publicado: 03/01/2022

Giovanni Ferreira Santos
ORCID: https://orcid.org/0000-0001-6275-0892
Centro Universitário de Patos de Minas, Brasil
E-mail: giovanniferreira @ unipam.edu.br
Guilherme de Queiroz Nunes e Silva
ORCID: https://orcid.org/0000-0001-8158-1510
Centro Universitário de Patos de Minas, Brasil
E-mail: guilhermeqnes @unipam.edu.br
Douglas Ribeiro Moreira
ORCID: https://orcid.org/0000-0003-3257-8643
Centro Universitário de Patos de Minas, Brasil
E-mail: douglasmoreira@ unipam.edu.br
Bruno Gontijo Vergutz
ORCID: https://orcid.org/0000-0002-6011-446X
Centro Universitário de Patos de Minas, Brasil
E-mail: brunovergutz@ unipam.edu.br
João Pedro de Miranda Carvalho
ORCID: https://orcid.org/0000-0001-8313-2903
Centro Universitário de Patos de Minas, Brasil
E-mail: joaocarvalho1 @unipam.edu.br
João Pedro Arruda Pessoa
ORCID: https://orcid.org/0000-0002-4869-203X
Centro Universitário de Patos de Minas, Brasil
E-mail: joaoarruda@ unipam.edu.br
Valter Paz do Nascimento Junior
ORCID: https://orcid.org/0000-0003-4498-2811
Centro Universitário de Patos de Minas, Brasil
E-mail: valterpj@ @unipam.edu.br
Natália Filardi Tafuri
ORCID: https://orcid.org/0000-0002-8445-0521
Centro Universitário de Patos de Minas, Brasil
E-mail: nataliaft@ unipam.edu.br

\section{Resumo}

Introdução: A Doença de Parkinson (DP) está entre os distúrbios neurológicos que mais crescem em todo o mundo, caracterizando-se como a segunda doença neurodegenerativa mais prevalente. No Brasil, a notificação da DP não é obrigatória, o que dificulta a estimativa de sua prevalência no país. Objetivo: Fazer o delineamento do perfil epidemiológico de internações relacionadas à DP no Brasil, associando faixa etária, sexo dos indivíduos acometidos com DP, tempo, caráter (urgente e eletivo) e gastos da internação. Metodologia: Tratou-se de um estudo epidemiológico descritivo, retrospectivo com abordagem quantitativa, sendo os dados obtidos por meio de consulta ao Departamento de Informática do Sistema Único de Saúde (DATASUS). A população de interesse foi indivíduos com DP, notificados no Brasil entre janeiro de 2016 até dezembro de 2020, com foco entre 60 e 89 anos. Resultados e Discussão: Verificou-se ente 2016 e 2020, o maior número de internações de indivíduos com DP ocorreu com pacientes de 60 a 79 anos, sendo o sexo masculino mais acometido pelo número de internações e óbitos. O sudeste mostrou maior número de internações e o nordeste maior média de permanência. O total de óbitos foi 281, gerando custos de 19 milhões de reais. Conclusão: Os dados obtidos revelaram padrões que seguem estáveis ao longo dos anos, de acordo com a região, sexo, óbitos e a faixa etária, embora existam possíveis problemas com subnotificações e escassez de informações. Assim, fica claro a necessidade de mais estudos para delineamentos futuros dos padrões epidemiológicos da DP no Brasil.

Palavras-chave: Doença de Parkinson; Epidemiologia; Hospitalização.

\section{Abstract}

Introduction: Parkinson's Disease (PD) is among the fastest growing neurological disorders in the world, characterized as the second most prevalent neurodegenerative disease. In Brazil, notification of PD is not mandatory, which makes it difficult to estimate its prevalence in the country. Objective: To outline the epidemiological profile of 
hospitalizations related to PD in Brazil, associating age group, sex of individuals affected with PD, time, character (urgent and elective) and hospitalization costs. Methodology: This was a descriptive, retrospective epidemiological study with a quantitative approach, with data obtained through consultation with the Department of Informatics of the Unified Health System (DATASUS). The population of interest was individuals with PD, reported in Brazil between January 2016 and December 2020, with a focus between 60 and 89 years old. Results and Discussion: It was found between 2016 and 2020, the highest number of admissions of individuals with PD occurred with patients aged 60 to 79 years, with males being more affected by the number of admissions and deaths. The Southeast showed the highest number of hospitalizations and the Northeast the highest average length of stay. The total number of deaths was 281 , generating costs of 19 million reais. Conclusion: The data obtained revealed patterns that remain stable over the years, according to region, gender, deaths and age group, although there are possible problems with underreporting and lack of information. Thus, the need for further studies for future delineations of the epidemiological patterns of PD in Brazil is clear.

Keywords: Parkinson's Disease; Epidemiology; Hospital admission.

\section{Resumen}

Introducción: La enfermedad de Parkinson (EP) se encuentra entre los trastornos neurológicos de más rápido crecimiento en el mundo, caracterizada como la segunda enfermedad neurodegenerativa más prevalente. En Brasil, la notificación de la EP no es obligatoria, lo que dificulta estimar su prevalencia en el país. Objetivo: Esbozar el perfil epidemiológico de las hospitalizaciones relacionadas con la EP en Brasil, asociando grupo de edad, sexo de los afectados por la EP, tiempo, carácter (urgente y electivo) y costos de hospitalización. Metodología: Estudio epidemiológico descriptivo, retrospectivo, con abordaje cuantitativo, con datos obtenidos mediante consulta con el Departamento de Informática del Sistema Único de Salud (DATASUS). La población de interés fueron individuos con EP, reportados en Brasil entre enero de 2016 y diciembre de 2020, con un enfoque entre 60 y 89 años. Resultados y Discusión: Se encontró que entre 2016 y 2020, el mayor número de ingresos de personas con EP ocurrió con pacientes de 60 a 79 años, siendo los hombres los más afectados por el número de ingresos y muertes. El sureste presentó el mayor número de hospitalizaciones y el noreste la mayor duración promedio de estadía. El número total de muertes fue de 281, generando costos de 19 millones de reales. Conclusión: Los datos obtenidos revelaron patrones que se mantienen estables a lo largo de los años, según región, género, defunciones y grupo de edad, aunque existen posibles problemas de subregistro y falta de información. Por lo tanto, es clara la necesidad de más estudios para delinear en el futuro los patrones epidemiológicos de la EP en Brasil.

Palabras clave: Enfermedad de Parkinson; Epidemiología; Hospitalización.

\section{Introdução}

A Doença de Parkinson (DP) está entre os distúrbios neurológicos que mais crescem em todo o mundo, caracterizando-se como a segunda doença neurodegenerativa mais prevalente (Bovolenta \& Felicio, 2016; Dorsey \& Sherer et al., 2018; Simon et al., 2019). Expectativas mostram que portadores de DP possam chegar a 17 milhões até 2040, impulsionados pelo aumento da longevidade, o declínio das taxas de tabagismo e o aumento da industrialização (Dorsey \& Elbaz et al., 2018). No Brasil, a notificação da DP não é obrigatória, o que dificulta a estimativa de sua prevalência no país. Porém segundo o IBGE, surgem 36 mil novos casos por ano, estimando-se uma prevalência atual com certa de 200 mil indivíduos com DP, sendo que em pessoas com 60 e 69 anos é de 700/100.000 casos, e entre 70 e 79 anos é de 1500/100.000 casos (IBGE, 2000).

A DP foi descrita pela primeira vez, por James Parkinson, em 1817, a qual é expressa de forma crônica e progressiva, com causa idiopática (Correia et al., 2013). As causas que desencadeiam as manifestações do processo neurodegenerativo da doença não estão totalmente elucidadas e compreendidas, porém acredita que é resultado, principalmente, da morte dos neurônios produtores de dopamina acarretando assim, na diminuição da transmissão dopaminérgica nos gânglios da base (disfunção dopaminérgica), cruzando também, com efeitos monoaminérgicos, o que afetaria os sistemas colinérgicos, serotoninérgicos e noradrenérgicos (Souza et al., 2011; Correia et al., 2013).

As manifestações neurodegenerativas provocados pela doença apresentam sinais típicos como: rigidez, tremor, bradicinesia e instabilidade postural (marcha lenta, queda e postura com a cabeça e tronco fletidos, com dificuldade para se ajustar à postura em inclinações súbitas). Além disso, disfunções cognitivas, alterações de sensibilidade, incontinência urinária, 
disfunção sexual, distúrbio do sono e reflexos profundos hiporresponsivos também são encontrados (Souza et al., 2011). Essas alterações desencadeiam a diminuição dos movimentos voluntários, incapacitando e limitando o portador por toda a vida.

A DP ainda não apresenta cura, porém, o tratamento convencional tem como foco a diminuição dos sintomas presentes na patologia (Dorsey \& Sherer et al., 2018). A primeira opção de tratamento consiste na administração de levodopa (L-dopa), mas outros fármacos também são utilizados, dentre eles a amantadina, anticolinérgicos, inibidores da monoamina oxidase B (MAO-B) e agonistas dopaminérgicos (Tosta et al., 2010).

No geral o tratamento é conservador, sendo que algumas condutas são avaliadas como outras opções de tratamento, como por exemplo a utilização de medicamentos com função neuroprotetora, não existindo, entretanto, relatos que comprovem evidências suficientes para o uso corrente de uma droga antiparkinsoniana com ação neuroprotetora (Ferreira-Junior et al., 2019). Nesse contexto, estratégias de tratamento para DP se tornam algo de grande valia para o atual cenário epidemiológico da doença, uma vez que, os portadores de DP são pacientes que precisam de medicamentos para o resto de suas vidas, utilizam muito os serviços de saúde, sendo mais propensos à internação em hospitais devido à doença ou outros fatores correlacionados, além de precisarem de cuidados e adaptações domiciliares para sua conveniência e segurança (Bovolenta \& Felicio, 2016).

Portanto, o objetivo desse trabalho foi fazer o delineamento do perfil epidemiológico de internações relacionadas à DP no Brasil, associando faixa etária, sexo dos indivíduos acometidos com DP, tempo, caráter (urgente e eletivo) e gastos da internação.

\section{Metodologia}

Tratou-se de um estudo epidemiológico descritivo, retrospectivo com abordagem de natureza quantitativa (Gil, 2002). Os dados foram obtidos por meio de consulta ao Departamento de Informática do Sistema Único de Saúde (DATASUS), no endereço eletrônico (http://www.datasus.gov.br), através do acesso às bases de dados de assistência à saúde e indicadores hospitalares. O levantamento de dados ocorreu no período de novembro a dezembro de 2021. Para evitar erros de retardo de notificação, optou-se por analisar os dados disponíveis de janeiro de 2016 até dezembro de 2020, último ano em que constavam os dados completos.

Nesse período, a análise foi feita sob toda a faixa etária que recebeu o diagnóstico da DP, segundo a Classificação Internacional de Doenças (CID-10), porém o presente artigo teve foco no grupo que se concentra dos 60 aos 89 anos. Assim, foram analisados os números de internações, o gasto nacional de acordo com o as internações, o caráter de atendimento, a média de permanência de internação desses pacientes e o número de óbitos.

Os dados foram inicialmente tabulados no Microsoft Office Excel $^{\circledR} \mathrm{e}$ foram tratados estatisticamente (em termos de média, correlações e números absolutos), por meio do programa Statistical Package for the Social Sciences (SPSS) versão 20.0. Por se tratar de um banco de domínio público, não foi necessário submeter o projeto ao Comitê de Ética em Pesquisa.

\section{Resultados}

Ao considerar o estudo proposto, os dados coletados via DataSUS/TabNet apontam que a DP correspondeu no período de 2016 a 2020, como o quarto motivo de hospitalizações por doenças que afetam o Sistema Nervoso Central (Capítulo 6 - CID-10).

Nesse período, foram observadas 4637 internações decorrentes da DP. A região com maior número de internação foi a região Sudeste (2.283 internações), seguida da região Sul (1.294 internações) e a que apresenta menor número de internação foi a região Norte (155 internações). O maior número de internação a nível nacional ocorreu no ano de 2016 (1140 internações) e o menor no ano de 2020 (592 internações). Além disso, ficou evidente um padrão no número de internações ao direcionar a 
atenção para o ano da internação, isso evidencia certa estagnação dos tratamentos. É interessante observar a ruptura desse padrão em 2020 (Tabela 1).

Tabela 1 - Número de internações decorrentes da DP, no Brasil, entre 2016 e 2020.

\begin{tabular}{|c|c|c|c|c|c|c|c|c|}
\hline \multirow{2}{*}{ Ano } & & \multicolumn{5}{|c|}{ Região } & \multicolumn{2}{|c|}{ Total } \\
\hline & & Norte & Nordeste & Sudeste & Sul & Centro-Oeste & $\mathrm{N}$ & $\%$ \\
\hline 2015 & & 4 & 10 & 39 & 19 & 4 & 76 & 1,64 \\
\hline 2016 & & 29 & 179 & 561 & 296 & 75 & 1140 & 24,58 \\
\hline 2017 & & 28 & 121 & 464 & 276 & 49 & 938 & 20,23 \\
\hline 2018 & & 29 & 102 & 469 & 252 & 76 & 928 & 20,01 \\
\hline 2019 & & 47 & 121 & 476 & 260 & 59 & 963 & 20,77 \\
\hline 2020 & & 18 & 82 & 274 & 191 & 27 & 592 & 12,77 \\
\hline & $\mathrm{N}$ & 155 & 615 & 2283 & 1294 & 290 & & \\
\hline Total & $\%$ & 3,34 & 13,26 & 49,23 & 27,91 & 6,25 & 4637 & 100,00 \\
\hline
\end{tabular}

Fonte: Ministério da Saúde - Sistema de Informações Hospitalares do SUS (SIH/SUS), 2021.

Em relação às características sociodemográficas dos indivíduos com DP internados, a maioria era do gênero masculino (59,69\%), com idade superior aos 50 anos, sendo aqueles com faixa etária entre 60 e 79 anos $(52,45 \%)$ os mais prevalentes, enquanto a menor faixa etária observada foi indivíduos de 19 anos $(0,38 \%)$ seguidos daqueles entre 20 a 29 anos $(0,72 \%)$ (tabela 2).

Na Tabela 3, observa-se que o número médio de dias de internação de indivíduos com diagnóstico de DP, por idade, aumenta de forma proporcional ao aumento da idade. De forma especial, indivíduos com mais de 80 anos, permaneceram aproximadamente por 18 dias na Atenção Hospitalar, período bem maior ao comparar com período de internação de indivíduos entre 40 a 49 anos (média 7,4 dias). Ademais, pela tabela 4, observa-se, também, uma disparidade entre a média de permanência hospitalar de acordo com a região do país, sendo que a maior média de permanência foi na região nordeste (18,3 dias) e as menores, na região norte $(7,6)$ e sul $(7,7)$

Tabela 2 - Número de internações por DP no Brasil, quanto à faixa etária e ao sexo, entre 2016 e 2020.

\begin{tabular}{ccccccc}
\hline \multirow{2}{*}{ Faixa Etária } & \multicolumn{2}{c}{ Masculino } & \multicolumn{2}{c}{ Feminino } & \multicolumn{3}{c}{ Total } \\
& $\mathrm{N}$ & $\%$ & $\mathrm{~N}$ & $\%$ & $\mathrm{~N}$ & $\%$ \\
\hline$\leq 19$ anos & 25 & 0,90 & 16 & 0,86 & 41 & 0,88 \\
20 a 29 anos & 24 & 0,87 & 10 & 0,54 & 34 & 0,73 \\
30 a 39 anos & 52 & 1,88 & 27 & 1,44 & 79 & 1,70 \\
40 a 49 anos & 201 & 7,26 & 130 & 6,96 & 331 & 7,14 \\
50 a 59 anos & 619 & 22,36 & 270 & 14,45 & 889 & 19,17 \\
60 a 69 anos & 767 & 27,71 & 443 & 23,70 & 1210 & 26,09 \\
70 a 79 anos & 710 & 25,65 & 511 & 27,34 & 1221 & 26,33 \\
80 anos e mais & 370 & 13,37 & 462 & 24,72 & 832 & 17,94 \\
\hline Total & 2768 & 100,00 & 1869 & 100,00 & 4637 & 100,00 \\
\hline
\end{tabular}

Fonte: Ministério da Saúde - Sistema de Informações Hospitalares do SUS (SIH/SUS), 2021. 
Tabela 3 - Média (dias) de permanência de internação hospitalar em indivíduos com DP, quanto à faixa etária, entre 2016 e 2020 .

\begin{tabular}{cc}
\hline Faixa Etária & Média permanência (dias) \\
\hline$\leq 19$ anos & 5,4 \\
20 a 29 anos & 4,9 \\
30 a 39 anos & 5,1 \\
40 a 49 anos & 7,4 \\
50 a 59 anos & 11,6 \\
60 a 69 anos & 10,5 \\
70 a 79 anos & 18 \\
80 anos e mais & 18,3 \\
\hline Total & 13,7 \\
\hline
\end{tabular}

Fonte: Ministério da Saúde - Sistema de Informações Hospitalares do SUS (SIH/SUS), 2021.

Tabela 4 - Média (dias) de permanência de internação hospitalar em indivíduos com DP, quanto a região e ao número de internações entre 2016 e 2020.

\begin{tabular}{ccc}
\hline Região & Internações (\%) & Media permanência (dias) \\
\hline Norte & $155(3,3 \%)$ & 7,6 \\
Nordeste & $615(12,2 \%)$ & 18,3 \\
Sudeste & $2.283(49,2 \%)$ & 17,0 \\
Sul & $1.294(27,9 \%)$ & 7,7 \\
Centro-Oeste & $290(6,25 \%)$ & 7,9 \\
\hline Total & $4.637(100 \%)$ & 13,7 \\
\hline
\end{tabular}

Fonte: Ministério da Saúde - Sistema de Informações Hospitalares do SUS (SIH/SUS), 2021.

Em relação ao número de óbitos por DP ocorrido no período da pesquisa, observou-se que o ano com maior registro de óbitos foi 2016, e que apesar da maior incidência da DP em homens não há grandes diferenças de óbitos em relação ao gênero, com percentual de 51,24\% para o sexo masculino e 48,76\% para o sexo feminino. Além disso, os óbitos acima de 60 anos foram responsáveis por $95 \%$ de todas ocorrências, não sendo observados óbitos abaixo dos 30 anos de idade (Tabela 5).

Tabela 5 - Número de óbitos decorrentes da DP, quanto a faixa etária e ao sexo, ente 2016 e 2020.

\begin{tabular}{ccccc}
\hline Faixa Etária & Masculino & Feminino & N & Total \\
\hline 30 a 39 anos & 1 & 1 & 2 & 0,71 \\
50 a 59 anos & 8 & 4 & 12 & 4,27 \\
60 a 69 anos & 37 & 15 & 52 & 18,51 \\
70 a 79 anos & 52 & 39 & 91 & 32,38 \\
80 anos e mais & 46 & 78 & 124 & 44,13 \\
Total & 144 & 137 & 281 & 100,00 \\
\hline
\end{tabular}

Fonte: Ministério da Saúde - Sistema de Informações Hospitalares do SUS (SIH/SUS), 2021. 
Em relação ao caráter de atendimento, $73,54 \%$ dos atendimentos foram caracterizados como eletivo, enquanto os outros $26,46 \%$ foram considerados de urgência, tendo um gasto total para os cofres públicos de mais de 19 milhões de reais, com um gasto médio anual de 3,8 milhões, sendo que os anos com os maiores gastos foram 2018 e 2019 (Tabela 6).

Tabela 6 - Gasto anual em relação ao total de internações em portadores da DP, entre 2016 e 2020.

\begin{tabular}{ccc}
\hline Ano processamento & Total de Internações & Valor total (R\$) \\
\hline $\mathbf{2 0 1 6}$ & 1154 & $3.763 .513,77$ \\
$\mathbf{2 0 1 7}$ & 939 & $3.634 .116,21$ \\
$\mathbf{2 0 1 8}$ & 921 & $4.260 .333,01$ \\
$\mathbf{2 0 1 9}$ & 947 & $4.514 .491,90$ \\
$\mathbf{2 0 2 0}$ & 676 & $2.966 .215,63$ \\
\hline Total & 4637 & $19.138 .670,52$ \\
\hline
\end{tabular}

Fonte: Ministério da Saúde - Sistema de Informações Hospitalares do SUS (SIH/SUS), 2021.

\section{Discussão}

Inicialmente é importante relatar que durante a pesquisa observou-se uma limitação decorrente da escassez de dados ofertados pelas plataformas gerenciada pelo DataSUS, visto que, a doença não é instrumento de notificação compulsória no país.

No que tange aos dados hospitalares em relação à DP, pode-se observar que a mortalidade, devido ao Parkinson, ainda é uma situação presente no país e que merece atenção, visto que, no intervalo entre 2016 e 2020 observou-se 4637 casos e 281 óbitos pela doença, sendo que a quantidade de internações e a média de permanência aumentam de maneira pronunciada de acordo com o avanço da idade, principalmente a partir dos 50 anos. Não foi observado de forma expressiva internações em indivíduos até os 50 anos de idade, algo já esperado, visto que registros de DP abaixo de 20 anos são caracterizados como Parkinson Juvenil e dos 20 aos 50 anos, são relativos à Doença de Parkinson Precoce, diagnósticos caracterizados por situações relativamente muito raras (Fahn, 2018).

Nessa perspectiva, o aspecto sociodemográfico mundial e nacional vem apresentando profundas alterações na pirâmide etária, ao considerar um parâmetro de envelhecimento médio da população. De acordo com o Programa das Nações Unidas para o Desenvolvimento (PNUD), o Brasil está intrinsicamente relacionado com as mudanças nos paradigmas populacionais, apresentando em 2014, um Índice de Desenvolvimento Humano (IDH) de 0,755. Ao comparar com a década anterior, em 2004 indicava um IDH de 0,698, resultando diretamente em melhorias gerais na qualidade de vida, fator esse, relacionado com maior longevidade e consequentemente aumento da incidência de doenças neurodegenerativas, como a DP (PNUD, 2014; Redone, 2021).

Ainda nesse contexto, os mesmos autores (PNUD, 2014; Redone, 2021) colocam que o Chile, em 2014 possuía IDH de 0,832 , indicando melhores parâmetros gerais, melhor qualidade de vida e maior longevidade quando comparado com o Brasil, enquanto Leiva (2019), em um estudo desenvolvido entre 1990 e 2016 indicou que a DP mostrou acréscimo de 19,9\% de prevalência de DP no Chile, destacando-se como o país latino-americano com o maior número de casos da patologia, enquanto o Brasil ocupa a sétima posição no que se refere à prevalência de DP. Isso sugere, que o aumento da incidência de DP pode estar diretamente relacionado ao aumento na expectativa de vida e índices avaliadores da qualidade de vida, como o IDH (PNUD, 2014; Redone, 2021).

A consequência direta da inversão da pirâmide etária (que está acontecendo no Brasil) propicia o surgimento de patologias associadas a processos neurodegenerativos, sendo a DP, a segunda enfermidade com maior incidência, afetando uma a cada 100 pessoas maiores de 60 anos (Alho, 2011; Leiva et al., 2019). Isso a nível global, proporciona um aumento de 
duas vezes na prevalência da DP nos últimos 26 anos, numericamente representados por 2,5 milhões de pessoas em 1990 para 6,1 milhões em 2016 acometidas com a patologia (Dorsey \& Elbaz et al., 2018). Essa evolução da patologia é destacada por uma prevalência com relação de 3:2 para homens em relação as mulheres, proporção que não é observada nas mortes, de acordo com os aspectos epidemiológicos brasileiros fornecidos pelo DataSUS (Fahn, 2018).

Outra explicação para os possíveis aumentos de casos e a etiologia da DP, estão relacionados aos hábitos de vidas. Acredita-se que a DP tem relação indireta com a redução do tabagismo, destacando um aumento da patologia de acordo com a queda das taxas de utilização da substância, ou seja, estudos associam que o aumento do risco na incidência de DP está relacionado com o abandono da prática tabagista, evidenciando o tabagismo como um fator protetor (Leiva et al., 2019). Outros estudos, associam esse aumento da DP com fatores externos como exposição à neurotoxinas ambientais, onde o paciente é exposto de maneira direta ou indireta a pesticidas e herbicidas, como, por exemplo, a toxina MPTP (1-Metil-4-Fenil-

\section{1,2,3,6-Tetrahidropiridina) (Santos, 2015).}

Ao observar a média de permanência hospitalar de pacientes com diagnóstico de DP, pode-se inferir uma análise do tratamento e dos efeitos desse na qualidade de vida do paciente. Em um estudo retrospectivo, analisou-se durante 5 anos, um grupo com 5637 pacientes com DP (média 70 anos) em comparação com 8143 pessoas no grupo controle (sem diagnóstico primário ou secundário de DP), evidenciando que os pacientes com DP têm elevada probabilidade de necessitarem de hospitalização, principalmente homens, com um tempo de hospitalização maior quando comparado com o grupo controle, com uma média de 7 dias a mais de internação. Dentre os motivos de maior hospitalização estiveram quedas, complicações motoras, problemas psiquiátricos, delirium, infecção geniturinária e efeitos adversos aos medicamentos (Lubomski et al., 2015; Fernandes \& Filho, 2018).

O tratamento pode ser subdividido em tratamento medicamentoso para sintomas motores, não motores, neuroprotetores, cirurgias e tratamentos alternativos e complementares para DP. A terapia medicamentosa busca a atenuação dos sintomas, de acordo com a progressão da doença, sendo que a terapia farmacológica é intensificada de maneira direta com a progressão da doença. Vale ressaltar que, com a progressão da idade, da neurodegeneração e do aumento dos sintomas, a terapia medicamentosa pode também proporcionar mais efeitos colaterais e, consequentemente, mais internações, destacandose a internação por depressão (Dong et al., 2018; Silva et al., 2021).

Para Dong et al., (2018) manejos alternativos e complementares ao tratamento farmacológico convencional da DP, contribuem não apenas para amenizar os sintomas, mas reduzir o tempo de permanência de hospitalizações. Entre essas condutas alternativas, estudos discutem o uso terapêutico do cannabidiol, que vem se mostrando bastante promissor como substância neuroprotetora, com menores efeitos colaterais em animais quando comparados com os atuais tratamentos, podendo contribuir futuramente, quem sabe, para um menor tempo de hospitalizações decorrentes de complicações próprias da DP ou de medicamentos usados para tratá-la (Dias-Tosta et al., 2010; Chagas et al., 2014; Crippa et al, 2019; Crippa, 2018; Peres et al, 2018).

Ainda considerando a importância da busca por novos tratamentos, uma vez que, entre os anos de 2016 a 2020, as 4.691 internações custaram aos cofres públicos mais de 18 milhões de reais, e considerando que a DP é uma afecção crônica, degenerativa e progressiva do sistema nervoso central, o uso de substâncias neuroprotetoras pode ser realmente uma alternativa relevante para a redução dos sintomas, número de hospitalizações e gasto financeiro (Souza et al., 2011; Lubomski et al., 2015).

Quanto à mortalidade, observou-se 281 óbitos pela DP, taxas essas, que parecem ser pequenas em relação ao período do tempo analisado. Entretanto, Benito-Leon (2018) salienta que os números de registro de óbito por DP podem apresentar uma grande subnotificação, de aspecto semelhante em todo o mundo, devido à baixa confiabilidade nos diagnósticos do óbito e ao fato da DP ser raramente apontada como causa básica nas declarações de óbito. Além disso, dados mundiais também 
coincidem com os aspectos observados no estudo, com informações dos Estados Unidos da América e dos países nórdicos de mortalidade quase desprezível abaixo dos 45 anos (no Brasil, entre 2016 e 2020 houve 0 mortes na faixa etária dos 40 aos 49 anos) e aumentadas com o avançar da idade, sendo a gravidade dos sintomas motores diretamente proporcional ao acontecimento do óbito, os quais poderiam ser atenuados com tratamentos alternativos (Benito-Leon, 2018).

Por fim, houve uma quebra do padrão linear nos registros relativos ao número de internações e ao valor gasto com o tratamento, no sentido descendente no ano de 2020. De acordo com a FioCruz e com o Observatório de Política e Gestão Hospitalar (OPGH-GovBr), o início da pandemia do Covid-19 em março de 2020 alterou diretamente o perfil de internações no SUS, devido aos efeitos da quarentena, isolamento social e política de prioridades de atendimento hospitalar. Esses dados, confirmados pelo DataSus, também puderam ser observados no padrão epidemiológico da DP. Trabalhos epidemiológicos futuros que observem os dados com a progressão dos efeitos da Covid-19 são necessários para atualizar esse padrão e analisar as influências dessa quebra de padrão (Albuquerque, 2020).

\section{Conclusão}

Verificou-se que durante o período pesquisado (2016-2020) o maior número de internações de indivíduos com DP ocorreu com pacientes de 60 a 79 anos, sendo o sexo masculino mais acometido pelo número de internações e óbitos, a região sudeste foi a que possuiu o maior número de internações e a nordeste com a maior média de permanência, com um total de 281 óbitos, gerando custos na ordem de 19 milhões de reais aos cofres públicos.

Estudos acerca da epidemiologia da DP são escassos e as fontes de dados do Brasil e do mundo, também possuem dados epidemiológicos que não retratam fielmente a realidade da doença, devido, principalmente, às subnotificações. Com isso, esses fatos limitam e dificultam a descrição mais precisa e real da epidemiologia da DP no Brasil e no mundo.

Sendo assim, esse trabalho corrobora para que novos estudos epidemiológicos e experimentais acerca do cenário epidemiológico, fisiopatologia e alternativas terapêuticas para a DP possam surgir, visto que a doença tem um grande impacto financeiro e social na população, caracterizando como um problema de saúde pública que tende a agravar-se cada vez mais com o aumento da expectativa de vida mundial e a inversão da pirâmide etária no Brasil.

\section{Referências}

Alho, A. T. (2011). Caracterização da substância negra humana durante o envelhecimento. Faculdade de Medicina da Universidade de São Paulo.

Albuquerque, C. (2020). Pandemia diminui número e muda perfil de internações no SUS em 2020. Observatório de Política e Gestão Hospitalar. https://www.observatoriohospitalar.fiocruz.br/debates-e-opinioes/pandemia-diminui-numero-e-muda-perfil-de-internacoes-no-sus-em-2020.

Benito-León, J. (2018). Epidemiología de la enfermedad de Parkinson em España y su contextualizacíon mundial. Revista de Neurología. 66 (4).

Borges, V. \& Tosta, E. (2010). Recomendações para o tratamento da fase inicial da doença de Parkinson. Doença de Parkinson: recomendações. 3, $19-37$.

Bovolenta, T. M. \& Felício, A. C. (2016). Parkinson's patients in the Brazilian Public Health Policy Context. Editorial - Hospital Israelita Albert Einstein.

Brasil, M. S. (2021). Ministério da Saúde DATASUS. Banco de dados do Sistema Único de Saúde-DATASUS. https://datasus.saude.gov.br/informacoes-desaude-tabnet/.

Brasil, M. S. (2021). Ministério da Saúde DATASUS Faixa Etária. Banco de dados do Sistema Único de Saúde-DATASUS. http://tabnet.datasus.gov.br/cgi/tabcgi.exe?sih/cnv/niuf.def.

Brasil, M. S. (2021). Ministério da Saúde DATASUS Valor Total. Banco de dados do Sistema Único de Saúde-DATASUS. http://tabnet.datasus.gov.br/cgi/tabcgi.exe?sih/cnv/niuf.def.

Brasil, M. S. (2021). Ministério da Saúde DATASUS Caráter de Atendimento. Banco de dados do Sistema Único de Saúde-DATASUS. http://tabnet.datasus.gov.br/cgi/tabcgi.exe?sih/cnv/niuf.def.

Chagas, N. H. M., Zuardi, W. A., Tumas, V., Pena-Pereira, M. A., Sobreira, T. E., Bergamaschi, M. M., dos Santos, A. C., Teixeira, A. L., Hallak, J. E. C. \& Crippa, J. A. S. (2014) Effects of cannabidiol in the treatment of patients with Parkinson's disease: An exploratory double-blind trial. Journal of Psychopharmacology. 28(11), 1088-192. 
Crippa, S. A. J., Hallak J. E. C., Zuardi, A. W., Guimarães, F. S., Tumas, V. \& dos Santos, R. G. (2019). Is cannabidiol the ideal drug to treat non-motor Parkinson's disease symptoms?. European Archives of Psychiatry and Clinical Neuroscience. 269, 121-133.

Crippa, J. A., Guimarães, F. S., Campos, A. C. \& Zuardi, A. W. (2018). Translational investigation of the therapeutic potential of cannabidiol (CBD): Towards a new age. Frontiers in Imumunology. 9.

Dong, J., Cui, Y., Li, S. \& Le, W. (2016) Current Pharmaceutical Treatments and Alternative Therapies of Parkinson's Disease. Current Neuropharmacology. 14(4).

Dorsey, E. R., Sherer, T., Okun, M. S. \& Bloem, B. R. (2018). The Emerging Evidence of the Parkinson Pandemic. Journal of Parkinson's Disease. 8, 3-8.

Dorsey, E. R. \& Elbaz, A. (2018) Global, regional, and national burden of Parkinson's disease, 1990-2016: a systematic analysis for the Global Burden of Disease Study 2016. Lancet Neurology. 17. 939-53.

Embrapa Territorial (2020). Sistema de Inteligência Territorial Estratégica da Macrologística Agropecuária brasileira (SITE-MLog). Campinas. www.embrapa.br/macrologistica.

Fahn, S. \& Kang, J. (2018). Doença de Parkinson. In: Louis D. Merrit - Tratado de Neurologia. (13a ed.) 83, $702-720$.

Fernandes, I. \& Filho, A. S. (2018). Estudo Clínico-Epidemiológico de Pacientes com Doença de Parkinson em Salvador-Bahia. Revista Brasileira de Neurologia e Psiquiatra, 22(1).

Ferreira-Junior, N. C., Campos, A. C., Guimarães, F. S, Del-Bel, E., Zimmermann, P. M. R., Junior, L. B., Hallak, J. E., Crippa, J. A. \& Zuardi, A. W. (2020). Biological bases for a possible effect of cannabidion in Parkinson's disease. Brazilian Journal of Psychiatry, 42(2), 218-224.

Gil, A. C. (2002). Como elaborar projetos de pesquisa. 4. ed. São Paulo: Atlas.

IBGE - Instituto Brasileiro De Geografia E Estatística (2020). Censo Brasileiro de 2010.

Lubomski, M. Rushworth, R. L. \& Tisch, S. (2015). Hospitalisation and comorbidities in Parkinson's disease: a large Australian retrospective study. Journal of Neurology, Neurosurgery \& Psychiatry, 86, 324-330.

Leiva, A. M., Martinez-Sanguinetti, M. A. M., Troncoso-Pantoja, C. Nazar, G., Petermann-Rocha, F. \& Celis-Morales, C. (2019). Chile lidera el ranking latino americano de prevalencia de enfermedad de Parkinson. Revista de Medicina Chile. 147(4).

Peres, F. F., Lima, A. C., Hallak, J. E. C., Crippa, J. A., Silva, R. H. \& Abílio, V. C. (2018). Cannabidiol as a Promising Strategy to treat and Prevent Movement Disorders?. Frontiers in Pharmacology. 9, 482.

PNUD, Programa das Nações Unidas para o Desenvolvimento (2021). Ranking IDH Global 2014. www.br.undp.org/content/brazil/pt/home/idh0/rankings/idhglobal.html.

Dias-Tosta, E., Rieder, C. R. M., Borges, V. \& Neto, Y. C. (2010). Recomendações para o tratamento da doença de Parkinson. In: TOSTA, E. et al. Doença de Parkinson: recomendações. 1(2), 13-18.

Redone, Registros Brasileiros de Doenças Neurológicas (2021). Academia Brasileira de Neurologia: Registros Brasileiros de Doenças Neurológicas www.abneuro.org.br/redone.

Santos, V. L. (2015). Perfil epidemiológico da doença de Parkinson no Brasil. Centro Universitário de Brasília: Faculdade de Ciências da Educação E Saúde.

Silva, A. B. G. (2021). Doença de Parkinson: revisão de literatura. Revista Brazilian Journal of Development, 7(4).

Simon, D. K., Tanner, C. M. \& Brundin, P. (2019). Parkinson Disease Epidemiology, Pathology, Genetics, and Pathopshysiology. Clinics in geriatric medicine. 36(1), 1-12.

Souza, C. F. M., Almeida, H. C. P., Sousa, J. B., Costa, P. H., Silveira, Y. S. S. \& Bezerra, J. C. L. (2011). A Doença de Parkinson e o Processo de Envelhecimento Motor: Uma Revisão de Literatura. Revista Neurociências, 14(4). 